November 2002

\title{
Mixed phases of color superconducting quark matter
}

\author{
F. Neumann ${ }^{a}$, M. Buballa ${ }^{a, b}$, and M. Oertel ${ }^{c}$ \\ ${ }^{a}$ Institut für Kernphysik, TU Darmstadt, Schlossgartenstr. 9, 64289 Darmstadt, Germany \\ ${ }^{b}$ Gesellschaft für Schwerionenforschung (GSI), Planckstr. 1, 64291 Darmstadt, Germany \\ c IPN-Lyon, 43 Bd du 11 Novembre 1918, 69622 Villeurbanne Cédex, France
}

\begin{abstract}
We examine electrically and color neutral quark matter in $\beta$-equilibrium focusing on the possibility of mixed phases between different color superconducting phases. To that end we apply the Gibbs criterion to ensure phase equilibrium and discuss the external conditions under which these mixed phases can occur. Neglecting surface and Coulomb effects we find a rich structure of different mixed phases with up to four components, including 2SC and CFL matter as well as more "exotic" components, like a phase with $u s$ - and $d s$-pairing but without $u d$-pairing. Preliminary estimates indicate, however, that the mixed phases become unstable if surface and Coulomb effects are included.
\end{abstract}

\section{Introduction}

The possible existence of color superconducting matter in the core of a neutron star is subject to intensive discussions since it has been discovered [1, 2] that at intermediate densities the energy gaps can be of the order of $100 \mathrm{MeV}$. These large values have been obtained in the scalar color antitriplet channel where instanton interactions [3] as well as interactions derived from single-gluon exchange predict the largest attraction. There are two different condensation patterns in this channel, depending on whether or not the strange quarks, which are more massive than the light up and down quarks, participate in forming a condensate,

$$
s_{A A^{\prime}}=\left\langle\psi^{T} C \gamma_{5} \tau_{A} \lambda_{A^{\prime}} \psi\right\rangle .
$$

Here both, $\tau_{A}$ and $\lambda_{A^{\prime}}$ are the antisymmetric generators of $S U(3)$, i.e., the antisymmetric Gell-Mann matrices $\left(A, A^{\prime} \in\{2,5,7\}\right)$, acting in flavor and color space, respectively. In 
the two-flavor color superconducting phase (2SC) where only the light quarks are involved in the condensation, the flavor index in Eq. (1) is restricted to $A=2$. In this case it is always possible, without loss of generality, to perform a color rotation such that the $2 \mathrm{SC}$ phase is described by $s_{22} \neq 0$ and $s_{A A^{\prime}}=0$ if $\left(A, A^{\prime}\right) \neq(2,2)$. The color-flavor-locked $(\mathrm{CFL})$ phase [4] is characterized by nonvanishing condensates $s_{22}, s_{55}$, and $s_{77}$, i.e., it contains $u d$ as well as $u s$ and $d s$ pairs. In the limiting case of equal masses and chemical potentials for all quarks these three condensates are equal, whereas in general they can be different from each other.

Most of the calculations so far have been performed using a common chemical potential for all quarks (see, e.g., [5, 6]). It has been found that in that case a large region of the phase diagram is occupied by a 2SC phase [7, 8]. For the interior of neutron stars, it is however important, to consider electrically and color neutral matter in $\beta$-equilibrium. As pointed out by Alford and Rajagopal this strongly disfavors or even rules out the $2 \mathrm{SC}$ phase in compact stars [10]. For example consider a system of massless up and down quarks together with electrons, but without strange quarks. Since the density of electrons is small (see, e.g., [11]), to achieve electric neutrality the density of $d$-quarks must be almost twice as large as the density of $u$-quarks, and hence $\mu_{d} \approx 2^{1 / 3} \mu_{u}$. This means that, e.g., for $\mu_{u}=400 \mathrm{MeV}$, the Fermi momenta of $u$ and $d$ differ by about $100 \mathrm{MeV}$, making $u d$ BCS-pairing very difficult. The presence of negatively charged strange quarks therefore is likely to favor pairing even if the strange quark mass is considerably larger. Alford and Rajagopal tackled that problem, performing an expansion in terms of the strange quark mass. They found that, whenever the 2SC phase is more favored than no pairing at all, the CFL phase is even more favored.

Recently this has been reinvestigated by Steiner, Reddy and Prakash [12], who analyzed electrically and color neutral quark matter within an NJL-type model, taking into account density dependent quark masses self-consistently. For those conditions which are relevant for neutron stars older than a few minutes, i.e., cold matter without trapped neutrinos, the authors found the $2 \mathrm{SC}$ phase to be most favored within a small region of quark number chemical potentials. They claim, however, that this region is likely to disappear, once the hadronic phase is properly taken into account [12].

The analyses of Refs. [10, 12] are based on a comparison of homogeneous phases: For a given quark number chemical potential $\mu$ the authors search for the phase which maximizes the pressure after the constraints of electrical and color neutrality have been imposed. The latter is achieved by introducing an additional chemical potential $\mu_{Q}$ which is related to electric charge and two chemical potentials $\mu_{3}$ and $\mu_{8}$ which are related to color. The values of these new chemical potentials needed to neutralize matter for a fixed value of $\mu$ depend on the phase. For instance the normal phase, where color invariance is not spontaneously broken, is color neutral for $\mu_{3}=\mu_{8}=0$, whereas a nonvanishing value of $\mu_{Q}$ is needed for electric neutrality. The situation is quite opposite to the CFL phase where $\mu_{8} \neq 0$ and $\mu_{Q}=\mu_{3}=0$ are needed [12]. Finally, in the 2SC phase both, $\mu_{8}$ and $\mu_{Q}$, must be nonzero. Hence neutral matter in one phase is never in chemical equilibrium

*Strictly speaking, color neutrality is not sufficient, but color singletness has to be imposed. This does, however, not induce a large cost in energy [9, such that we can consider matter which is only color neutral. 
with neutral matter in a different phase, even if their quark number chemical potentials $\mu$ are the same. In particular the points of equal pressure do not fulfill the Gibbs criteria for a phase transition stating that the pressure and all chemical potentials must be the same in coexisting phases.

One possibility to resolve this problem is to give up the requirement of separately neutral phases and to consider mixed phases of two components in chemical equilibrium which are only neutral in total. This procedure has been pushed forward by Glendenning in the context of the quark-hadron phase transition in neutron stars where a similar problem occurs 13. Since Glendenning did not consider color-superconducting phases, he only had to care about electric neutrality. In this case a neutral mixed phase can obviously be constructed in those regions of the phase boundary where the charge densities of the two components have opposite signs.

In the present paper we generalize this procedure to construct electrically and color neutral mixed phases. This is more difficult because for two charged and colored components a mixture which is electrically neutral is in general not color neutral and vice versa. In the four-dimensional space spanned by $\mu, \mu_{Q}, \mu_{3}$ and $\mu_{8}$ the phase boundaries are three-dimensional hypersurfaces. As we will show the regions where electrically and color neutral mixed phases are possible correspond to one-dimensional lines on these hypersurfaces.

Our calculations are performed within the same NJL type model which was the basis of the analysis of Ref. [12]. It includes interactions which allow for the condensation patterns described above as well as for quark-antiquark condensates $\langle\bar{\psi} \psi\rangle$. The latter, describing dynamical chiral symmetry breaking in the vacuum, in particular generate dynamically a constituent strange quark mass which can be of the order of the quark chemical potential [7]. We restrict ourselves to bulk quark matter in mean-field approximation. Of course if mixed phases are formed the domains belonging to a single component must have finite sizes determined by the electric and color-electric fields and the surface tension. In fact, if the latter is too large the mixed phase will become unstable and a single charged interface between the two phases will be more favorite. This scenario has been suggested for the transition from the hadronic phase to the CFL phase [14]. A crystalline color superconducting phase [15] in the transition region would be another possibility.

The paper is organized as follows: In Sec. 2 we introduce the conserved charges in our system and the related chemical potentials. We discuss the Gibbs condition for phase boundaries. Thereafter, in Sec. 3, we present the model which is used to describe quark matter. We continue in Sec. 1 with an examination of the resulting phase diagram in the space of the various chemical potentials. In Sec. 5 we construct electrically and color neutral mixed phases and discuss the resulting phase structure of neutral quark matter. Finally, in Sec. 6 we summarize and discuss some open questions related to our investigations. 


\section{Conserved charges, chemical potentials and phase transitions}

We consider a system of quarks, electrons and muons with the number densities $n_{f, c}, n_{e}$, and $n_{\mu}$, respectively. Here $f=u, d, s$ ("up", "down", "strange") refers to the flavor and $c=r, g, b$ ("red", "green", "blue") refers to the color. The total flavor and color densities are then given by

$$
n_{f}=\sum_{c} n_{f, c}, \quad n_{c}=\sum_{f} n_{f, c} .
$$

As mentioned in the Introduction we are mainly interested in describing the conditions present in compact stars older than a few minutes, when neutrinos can freely leave the system. In this case lepton number is not conserved and we have four independent conserved charges, namely the total electric charge

$$
n_{Q}=\frac{2}{3} n_{u}-\frac{1}{3} n_{d}-\frac{1}{3} n_{s}-n_{e}-n_{\mu}
$$

and the three color charges $n_{r}, n_{g}$, and $n_{b}$ or, equivalently, their linear combinations

$$
n=n_{r}+n_{g}+n_{b}, \quad n_{3}=n_{r}-n_{g}, \quad n_{8}=\frac{1}{\sqrt{3}}\left(n_{r}+n_{g}-2 n_{b}\right) .
$$

Here $n$ corresponds to the total quark number density, while $n_{3}$ and $n_{8}$ describe color asymmetries. Note that $n / 3$ also describes the conserved baryon number. The four conserved charges are related to four independent chemical potentials, $\mu, \mu_{3}, \mu_{8}$, and $\mu_{Q}$.

The individual chemical potentials $\mu_{f, c}$ "felt" by the quarks of flavor $f$ and color $c$ are then given by corresponding components of the diagonal matrix

$$
\hat{\mu}=\mu+\mu_{Q}\left(\frac{1}{2} \tau_{3}+\frac{1}{2 \sqrt{3}} \tau_{8}\right)+\mu_{3} \lambda_{3}+\mu_{8} \lambda_{8}
$$

Here, as before, $\tau_{i}$ and $\lambda_{j}$ are operators in flavor space and color space, respectively. The electron and muon chemical potentials are simply $\mu_{e}=\mu_{\mu}=-\mu_{Q}$. This implies

$$
\mu_{d, c}=\mu_{s, c}=\mu_{u, c}+\mu_{e} \text { for all } c
$$

which is usually referred to as $\beta$-equilibrium.

The total thermodynamic potential of our model is given by the sum of a quark part and a leptonic part,

$$
\Omega\left(T,\left\{\mu_{i}\right\} ; \chi\right)=\Omega_{q}\left(T,\left\{\mu_{i}\right\} ; \chi\right)+\Omega_{l}\left(T, \mu_{Q}\right)
$$

where $\left\{\mu_{i}\right\}=\left\{\mu, \mu_{3}, \mu_{8}, \mu_{Q}\right\}$. For the leptonic part we simply take a gas of noninteracting massless electrons and massive muons. The quark part will be further specified in Sec. 3 . Here we only note, that it depends on a set of condensates which we denote by $\chi$. In general there are different solutions for $\chi$ at given temperature and chemical potentials. Then the stable one is the solution which corresponds to the lowest value of $\Omega$. At 
the boundaries which separate two different phases the corresponding thermodynamic potentials coincide,

$$
\Omega\left(T,\left\{\mu_{i}\right\} ; \chi_{1}\right)=\Omega\left(T,\left\{\mu_{i}\right\} ; \chi_{2}\right),
$$

where $\chi_{1} \neq \chi_{2}$ if the phase transition is first order.

In a given phase the various densities defined above can be obtained from the thermodynamic potential as

$$
n_{i}=-\frac{\partial \Omega}{\partial \mu_{i}} .
$$

We are mostly interested in electrically and color neutral matter, which is characterized by

$$
n_{Q}=n_{3}=n_{8}=0 .
$$

For homogeneous phases the consequences of these constraints have been discussed in Refs. [10, 12]. The goal of the present article is to analyze possible mixed phases which could exist along first-order phase boundaries, Eq. (8).

Suppose we have a mixed phase consisting of two components, 1 and 2 . In general the densities $n_{i}^{(1)}$ and $n_{i}^{(2)}$ resulting from Eq. (9) will not be identical in both phases. In particular, in general Eq. (10) will not be fulfilled simultaneously for both components. However, as indicated in the Introduction it is sufficient to demand that the average charge and color densities of the mixed phase vanish [13]. If the two components occupy the volume fractions $x_{1}$ and $x_{2}=1-x_{1}$, respectively, the average densities are given by

$$
n_{i}=x_{1} n_{i}^{(1)}+\left(1-x_{1}\right) n_{i}^{(2)} .
$$

This is zero for

$$
x_{1}=\frac{n_{i}^{(2)}}{n_{i}^{(2)}-n_{i}^{(1)}} .
$$

To be meaningful the solution must be in the interval $0<x_{1}<1$. This is fulfilled when the charge densities $n_{i}^{(1)}$ and $n_{i}^{(2)}$ have opposite signs, which is an obvious prerequisite for a charge neutral mixture. For a single charge, e.g., $n_{Q}$, it is the only one. However, in order to get simultaneous neutrality for three charges, Eq. (10), we have to require that the result of Eq. (12) is the same for $i=Q, 3$, and 8. This is the case when

$$
n_{Q}^{(1)}: n_{3}^{(1)}: n_{8}^{(1)}=n_{Q}^{(2)}: n_{3}^{(2)}: n_{8}^{(2)}
$$

In our numerical calculations we will restrict ourselves to $T=0$, relevant for compact stars older than a few minutes. Then the phase boundaries, Eq. (8), are three-dimensional surfaces in the four-dimensional space spanned by $\mu, \mu_{Q}, \mu_{3}$, and $\mu_{8}$. Since Eq. (13) imposes two additional constraints, electrically and color neutral mixed phases can be constructed along a one-dimensional line. In the simplest case this line starts at a point where the neutrality line of phase $1\left(n_{Q}^{(1)}=n_{3}^{(1)}=n_{8}^{(1)}=0\right)$, meets the phase boundary and it ends where the neutrality line of phase 2 meets the phase boundary. Between these two points $x_{1}$ changes continuously from 1 to 0 . However, if the neutrality line meets another phase boundary before, one has three coexisting phases,

$$
\Omega\left(T,\left\{\mu_{i}\right\} ; \chi_{1}\right)=\Omega\left(T,\left\{\mu_{i}\right\} ; \chi_{2}\right)=\Omega\left(T,\left\{\mu_{i}\right\} ; \chi_{3}\right) .
$$


In this case the neutrality condition reads

$$
\hat{N} \vec{x} \equiv\left(\begin{array}{ccc}
n_{Q}^{(1)} & n_{Q}^{(2)} & n_{Q}^{(3)} \\
n_{3}^{(1)} & n_{3}^{(2)} & n_{3}^{(3)} \\
n_{8}^{(1)} & n_{8}^{(2)} & n_{8}^{(3)}
\end{array}\right)\left(\begin{array}{c}
x_{1} \\
x_{2} \\
x_{3}
\end{array}\right)=0
$$

In order to find a non-trivial solution for $\vec{x}$, we must have $\operatorname{det} \hat{N}=0$. Together with Eq. (14), this again restricts the possible solutions to a one-dimensional subspace. Moreover, since the fractions $x_{i}$ should not be negative, for each $m=Q, 3,8$ the densities $n_{m}^{(i)}$ must not have the same sign for all $i=1,2,3$.

Finally, there could even be a mixed phase, consisting of four components. The corresponding phase boundary is one-dimensional and again the region of possible neutral mixed phases is further restricted by the requirement that the various fractions $x_{i}$ should not be negative.

\section{Quark model}

The quark part of our model is defined by the Lagrangian

$$
\mathcal{L}_{e f f}=\bar{\psi}(i \not \supset-\hat{m}) \psi+\mathcal{L}_{q \bar{q}}+\mathcal{L}_{q q}
$$

where $\psi$ denotes a quark field with three flavors and three colors. The mass matrix $\hat{m}$ has the form $\hat{m}=\operatorname{diag}\left(m_{u}, m_{d}, m_{s}\right)$ in flavor space. Throughout this paper we will assume $m_{u}=m_{d}$, whereas the strange quark mass $m_{s}$ will be different. To study the interplay between the color-superconducting diquark condensates and the quark-antiquark condensates we consider an NJL-type interaction with a quark-quark part

$$
\mathcal{L}_{q q}=H \sum_{A=2,5,7} \sum_{A^{\prime}=2,5,7}\left(\bar{\psi} i \gamma_{5} \tau_{A} \lambda_{A^{\prime}} C \bar{\psi}^{T}\right)\left(\psi^{T} C i \gamma_{5} \tau_{A} \lambda_{A^{\prime}} \psi\right)
$$

and a quark-antiquark part

$\mathcal{L}_{q \bar{q}}=G \sum_{a=0}^{8}\left[\left(\bar{\psi} \tau_{a} \psi\right)^{2}+\left(\bar{\psi} i \gamma_{5} \tau_{a} \psi\right)^{2}\right]-K\left[\operatorname{det}_{f}\left(\bar{\psi}\left(1+\gamma_{5}\right) \psi\right)+\operatorname{det}_{f}\left(\bar{\psi}\left(1-\gamma_{5}\right) \psi\right)\right]$.

Here $\tau_{0}=\sqrt{\frac{2}{3}} \mathbb{1}_{f}$ is proportional to the unit matrix in flavor space. Eq. (18) corresponds to a typical 3-flavor NJL-model Lagrangian which has often been used to study meson spectra [16, 17] or properties of quark matter at finite densities or temperatures [18, 19, 11]. It consists of a $U(3)_{L} \times U(3)_{R}$-symmetric 4-point interaction and a 't Hooft-type 6-point interaction which breaks the the $U_{A}(1)$ symmetry. The above interaction terms might arise from some underlying more microscopic theory and are understood to be used at meanfield level in Hartree approximation. In particular we do not consider any contribution from the 6-point interaction to the diquark condensate. The same model has also been employed in Ref. [12] and for the case of one common chemical potential in Ref. [8]. 
In order to calculate the mean-field thermodynamic potential $\Omega_{q}$ at temperature $T$ and the various chemical potentials we linearize $\mathcal{L}_{\text {eff }}$ in the presence of the three quarkantiquark condensates $\phi_{f}=\langle\bar{f} f\rangle, f=u, d, s$, and the three diquark condensates $s_{A A}$, $A=2,5,7$. Introducing the constituent quark masses

$$
M_{i}=m_{i}-4 G \phi_{i}+2 K \phi_{j} \phi_{k}, \quad(i, j, k)=\text { any permutation of }(u, d, s),
$$

and the diquark gaps

$$
\Delta_{A}=-2 H s_{A A}
$$

and employing Nambu-Gorkov-formalism the result can be written in the following way:

$$
\begin{aligned}
\Omega_{q}\left(T,\left\{\mu_{i}\right\} ; \chi\right)= & -T \sum_{n} \int \frac{d^{3} p}{(2 \pi)^{3}} \frac{1}{2} \operatorname{Tr} \ln \left(\frac{1}{T} S^{-1}\left(i \omega_{n}, \vec{p}\right)\right) \\
& +2 G\left(\phi_{u}^{2}+\phi_{d}^{2}+\phi_{s}^{2}\right)-4 K \phi_{u} \phi_{d} \phi_{s}+H\left(\left|s_{22}\right|^{2}+\left|s_{55}\right|^{2}+\left|s_{77}\right|^{2}\right) .
\end{aligned}
$$

Here $\chi=\left\{\phi_{u}, \phi_{d}, \phi_{s}, s_{22}, s_{55}, s_{77}\right\}$ denotes the set of condensates. $S^{-1}$ is the inverse fermion propagator

$$
S^{-1}(p)=\left(\begin{array}{cc}
\not p-\hat{M}+\hat{\mu} \gamma^{0} & \Delta_{2} \gamma_{5} \tau_{2} \lambda_{2}+\Delta_{5} \gamma_{5} \tau_{5} \lambda_{5}+\Delta_{7} \gamma_{5} \tau_{7} \lambda_{7} \\
-\Delta_{2}^{*} \gamma_{5} \tau_{2} \lambda_{2}-\Delta_{5}^{*} \gamma_{5} \tau_{5} \lambda_{5}-\Delta_{7}^{*} \gamma_{5} \tau_{7} \lambda_{7} & \not p-\hat{M}-\hat{\mu} \gamma^{0}
\end{array}\right),
$$

where $\hat{M}=\operatorname{diag}\left(M_{u}, M_{d}, M_{s}\right)$. In Eq. (21), it has to be evaluated at $p=\left(i \omega_{n}, \vec{p}\right)$, where $\omega_{n}=(2 n-1) \pi T$ are fermionic Matsubara frequencies.

For the case of equal chemical potentials an explicit expression for $\Omega_{q}$ has been discussed in detail in Ref. [7]. For unequal chemical potentials this is more involved and we only state the general structure as far as necessary for our later discussion. As shown in Refs. [10, 12, 20] $S^{-1}$ can be decomposed into several blocks. Six of them have a $2 \times 2$ structure in the combined color-flavor space,

$$
\mathcal{M}_{f_{1} c_{1}, f_{2} c_{2}}=\left(\begin{array}{cc}
p_{f_{1}, c_{1}}^{+}-M_{f_{1}} & \Delta \gamma_{5} \\
-\Delta^{*} \gamma_{5} & p_{f_{2}, c_{2}}^{-}-M_{f_{2}}
\end{array}\right)
$$

where $p_{f, c}^{ \pm}=\not p \pm \mu_{f, c} \gamma_{0}$. These blocks describe pairing of two species of quarks with flavors $f_{1}$ and $f_{2}$ and colors $c_{1}$ and $c_{2}$, respectively. More precisely, one gets the following three combinations [12]: First, green up quarks are paired with red down quarks, where $\Delta=\Delta_{2}$, second, blue down quarks are paired with green strange quarks $\left(\Delta=\Delta_{5}\right)$, and third, red strange quarks are paired with blue up quarks $\left(\Delta=\Delta_{7}\right)$. The other three $2 \times 2$ blocks can be generated simply by interchanging the role of particles and antiparticles and lead to the same combinations.

The remaining contribution to $\Omega$ has the following $6 \times 6$-structure:

$$
\mathcal{M}_{u d s}=\left(\begin{array}{cccccc}
p_{u, r}^{+}-M_{u} & 0 & 0 & 0 & -\Delta_{2} \gamma_{5} & -\Delta_{5} \gamma_{5} \\
0 & p_{d, g}^{+}-M_{d} & 0 & -\Delta_{2} \gamma_{5} & 0 & -\Delta_{7} \gamma_{5} \\
0 & 0 & p_{s, b}^{+}-M_{s} & -\Delta_{5} \gamma_{5} & -\Delta_{7} \gamma_{5} & 0 \\
0 & \Delta_{2}^{*} \gamma_{5} & \Delta_{5}^{*} \gamma_{5} & p_{u, r}^{-}-M_{u} & 0 & 0 \\
\Delta_{2}^{*} \gamma_{5} & 0 & \Delta_{7}^{*} \gamma_{5} & 0 & p_{d, g}^{-}-M_{d} & 0 \\
\Delta_{5}^{*} \gamma_{5} & \Delta_{7}^{*} \gamma_{5} & 0 & 0 & 0 & \not p_{s, b}^{-}-M_{s}
\end{array}\right),
$$


involving red $u$-quarks, green $d$-quarks and blue $s$-quarks. This represents the particle and antiparticle contributions to the $3 \times 3$-block mentioned in Ref. [20].

As demonstrated by Rajagopal and Wilczek for a simplified two-quark model, the pairing of two quark species forces their densities to be equal [24]. Originally, it was expected that in the CFL phase, where all quarks are paired, all densities $n_{f, c}$ are equal. This would mean that the CFL phase is always electrically and color neutral. However, as pointed out by Steiner, Reddy, and Prakash [12], only those quarks which are paired in the same $2 \times 2$ block have the same density, whereas the densities could differ for different blocks. Furthermore the argument does not apply to the $6 \times 6$ block. For the CFL phase this means $n_{u, g}=n_{d, r}, n_{u, b}=n_{s, r}$, and $n_{d, b}=n_{s, g}$, and thus

$$
n_{u}=n_{r}, \quad n_{d}=n_{g}, \quad n_{s}=n_{b} \quad(\mathrm{CFL})
$$

This relation guarantees neutrality of CFL matter under the rotated electromagnetism $\tilde{Q}$ [10, 12], but in general it does not preclude the presence of ordinary electric or color charges. Note, however, that color neutral CFL matter is automatically electrically neutral as long as no leptons are present.

In the $2 \mathrm{SC}$ phase, where $\Delta_{5}=\Delta_{7}=0$, Eq. (24) can be further decomposed, and we obtain a new $2 \times 2$ block involving red $u$-quarks and green $d$-quarks. Together with the other $2 \times 2$ block which contains $\Delta_{2}$ this leads to the relations

$$
n_{u, r}=n_{d, g}, \quad n_{u, g}=n_{d, r} \quad(2 \mathrm{SC}) .
$$

The corresponding relations for other phases, e.g., with two nonvanishing diquark condensates, can be obtained analogously.

The further elaboration of the thermodynamic potential contains only straight forward manipulations, which will not be presented here. In Eq. (21) $\Omega_{q}$ depends on $\chi$, i.e., on the choice of the condensates $\phi_{f}$ and $s_{A A}$. In order to find the self-consistent solutions we have to look for the stationary points of the potential with respect to these condensates. As mentioned before, there is often more than one selfconsistent solution. In this case the stable solution is the one which minimizes the thermodynamic potential.

Our model is incomplete without specifying the parameters to be used in the numerical calculations. To this end we adopt the parameters of Ref. [19], which have been determined by fitting the pseudoscalar meson masses and decay constants in vacuum: a sharp 3momentum cutoff $\Lambda=602.3 \mathrm{MeV}$ to regularize the integrals, the bare quark masses $m_{u}=m_{d}=5.5 \mathrm{MeV}$ and $m_{s}=140.7 \mathrm{MeV}$, and the coupling constants $G=1.835 \Lambda^{-2}$ and $K=12.36 \Lambda^{-5}$. Similarly, one could fix the quark-quark coupling constant $H$ by fitting baryon masses within a Fadeev approach [21, 22]. For simplicity, however, we follow Ref. 12] (preprint versions v1 and v2) and take $H=G$. This is similar in magnitude to the value we have chosen in Ref. [7] where we took $H=3 / 4 G$, but with a larger $G$.

\footnotetext{
${ }^{\dagger}$ In the latest version of Ref. 12 (v3), the authors take $H=3 / 4 G$. Qualitatively, this does not change their results. Therefore, in the following we will not distinguish between the different versions. However, for any quantitative comparison with our results, the reader should refer to version v2.

${ }^{\ddagger}$ In Ref. [7] we used a four-point interaction with the quantum numbers of a single-gluon exchange,
} 

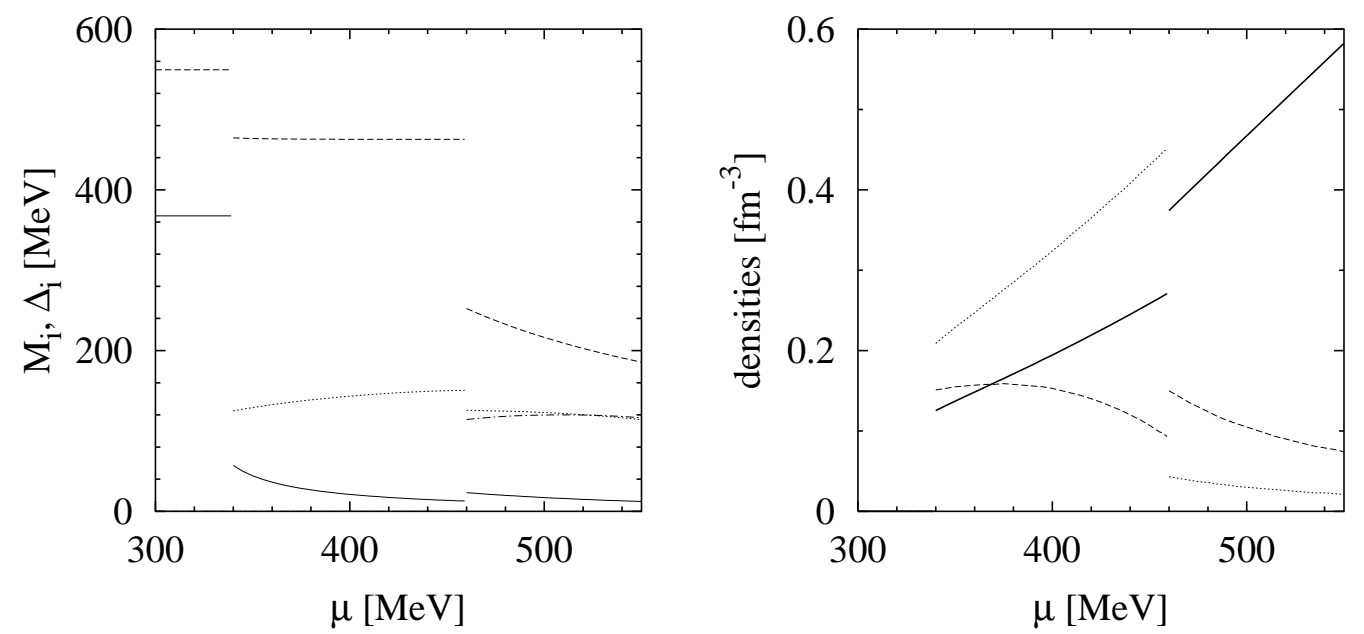

Figure 1: Gap parameters and densities at $T=\mu_{3}=\mu_{8}=\mu_{Q}=0$ as functions of $\mu$. Left: $M_{u}=M_{d}$ (solid), $M_{s}$ (dashed), $\Delta_{2}$ (dotted), and $\Delta_{5}=\Delta_{7}$ (dashed-dotted). Right: $n / 10$ (solid), $n_{8}$ (dashed), and $n_{Q}$ (dotted).

\section{Phase structure}

In order to construct the equation of state for electrically and color neutral quark matter (including possible mixed phases) we first have to explore the phase structure of the model and the charge densities as functions of the chemical potentials $\mu_{i}$.

We begin with the standard case of a single, color and flavor independent, chemical potential $\mu$ for all quarks, i.e., $\mu_{3}=\mu_{8}=\mu_{Q}=0$. For the parameters specified above we obtain the results which are displayed in Fig. 1. In the left panel the constituent masses and diquark gaps are shown. One can clearly distinguish three phases separated by first-order phase boundaries: At low chemical potentials we find a normal phase with vanishing diquark condensates, followed by the 2SC phase with $\Delta_{2} \neq 0$ and finally the CFL phase where also $\Delta_{5}$ and $\Delta_{7}$ are nonzero. At the phase boundaries we observe strong discontinuities in the quark masses [7, 8].

In the right panel we show the corresponding densities. Note that the quark number density $n$ (solid line) has been divided by 10 to fit to the scale. The dotted line corresponds to the electric charge density $n_{Q}$, the dashed line to the color density $n_{8}$. The color density $n_{3}$ is identically zero. As one can see, all densities vanish in the "normal phase", i.e., this phase corresponds to the vacuum. In fact, it has to be like this, because as soon as up and down quarks are present, their Fermi surfaces are subject to a Cooper instability leading to the formation of the diquark condensate $s_{22}$. (This argument will no longer go through, once we have switched on one of the other chemical potentials which lift the degeneracy

without six-point interaction. In this case a simple Fierz rearrangement yields $H: G=3: 4$. This relation also holds for a two-flavor instanton interaction. Hence, in our present model a simple estimate for the value of the diquark interaction, e.g., in the $u d$-channel, is given by $H=3 / 4 G_{\text {eff }}$, where $G_{\text {eff }}=$ $G-K\langle\bar{s} s\rangle$. In vacuum this is slightly larger than $G$, but it is a density dependent quantity and differs for different phases. Such effects have not been taken into account in our present work, but they are certainly worthwhile to be studied in future. 
of the Fermi surfaces of all up and down quarks.)

The two other phases carry both, electric and color charges. The electric charge of the 2SC phase is easily understood. Since $\mu_{Q}=0$, there are no leptons and the densities of up and down quarks are equal. Moreover, in our example there are no strange quarks, which are too heavy to be populated in this regime. Hence the total electric charge density is given by $n_{Q}=n / 6$. The nonvanishing color density $n_{8}$ reflects the fact that for equal chemical potentials the densities of the paired (red and green) quarks are larger than the density of the unpaired (blue) quarks [23]. Numerically we find $\left(n_{r}-n_{b}\right) / n=10 \%$ at the lower boundary and $\left(n_{r}-n_{b}\right) / n=3 \%$ at the upper boundary of the 2SC phase.

Just above the transition to the CFL phase this ratio does not change very much, whereas the electric charge density drops significantly due to a strong increase of the density of strange quarks. To a large extent, this is caused by a sudden drop of the strange quark mass, but this is only part of the story. For instance, at $\mu=500 \mathrm{MeV}$ we have $M_{u}=M_{d}=17.2 \mathrm{MeV}$ and $M_{s}=216.5 \mathrm{MeV}$. Using these numbers in a free gas approximation we would expect $n_{Q}=0.049 \mathrm{fm}^{-3}$, whereas numerically we find $n_{Q}=$ $0.030 \mathrm{fm}^{-3}$. This difference is caused by the diquark pairing, which links the flavor densities in the CFL phase directly to the color densities, as discussed in Eq. (25). For $n_{3}=0$ one finds $n_{Q}=1 /(2 \sqrt{3}) n_{8}$, in agreement with our numerical results.

Electrically and color neutral CFL and 2SC matter has been constructed in Ref. [12]. According to the charge densities $n_{8}$ and $n_{Q}$ which have to be removed, this in general requires the introduction of nonvanishing chemical potentials $\mu_{8}$ and $\mu_{Q}$. In order to see how these additional chemical potentials influence the phase boundaries, we first study them separately.

In Fig. 2 we show the phase diagram in the $\mu-\mu_{8}$-plane for $\mu_{Q}=\mu_{3}=0$. The (first-order) phase boundaries are indicated by solid lines. We find again the three phases discussed before, i.e., the normal phase, the 2SC phase, and the CFL phase. For $\mu_{8}=0$ we have seen that the "normal phase" actually corresponds to the vacuum. However, when $\mu_{b}=\mu-2 / \sqrt{3} \mu_{8}$ becomes larger than the vacuum masses of the light quarks (the region above the dashed line), blue up and down quark states can be populated forming a gas of unpaired blue quarks. Here we have neglected that in principle these quarks could pair in a different channel [25, 26]. In any case we should keep in mind that our model is not suited for a realistic description of the low-density regime, where confinement and hadronic degrees of freedom have to be taken into account.

For the color superconducting phases we have also indicated the lines of color neutral matter (dotted). As discussed in the previous section, in the CFL phase color neutral quark matter is automatically electrically neutral as well, i.e., in the CFL phase the dotted line corresponds to the neutral matter solution, which has been determined in Ref. [12]. It meets the phase boundary to the $2 \mathrm{SC}$ phase at $\mu=465.7 \mathrm{MeV}$ and $\mu_{8}=-32.5 \mathrm{MeV}$. The 2SC matter which is in chemical and mechanical equilibrium with the neutral CFL matter at this point carries both, electric and color charge, $n_{Q}=0.464 \mathrm{fm}^{-3}$ and $n_{8}=$ $-0.329 \mathrm{fm}^{-3}$. In the next section, this point will be the end point of the $2 \mathrm{SC}-\mathrm{CFL}$ mixed phase. Unlike color neutral CFL matter, color neutral 2SC matter is not electrically neutral but positively charged. In fact, a nonvanishing $\mu_{8}$ does not change the ratio of up and down quarks and hence, as long as no strange quarks are present, $n_{Q} / n=1 / 6$ as before. 


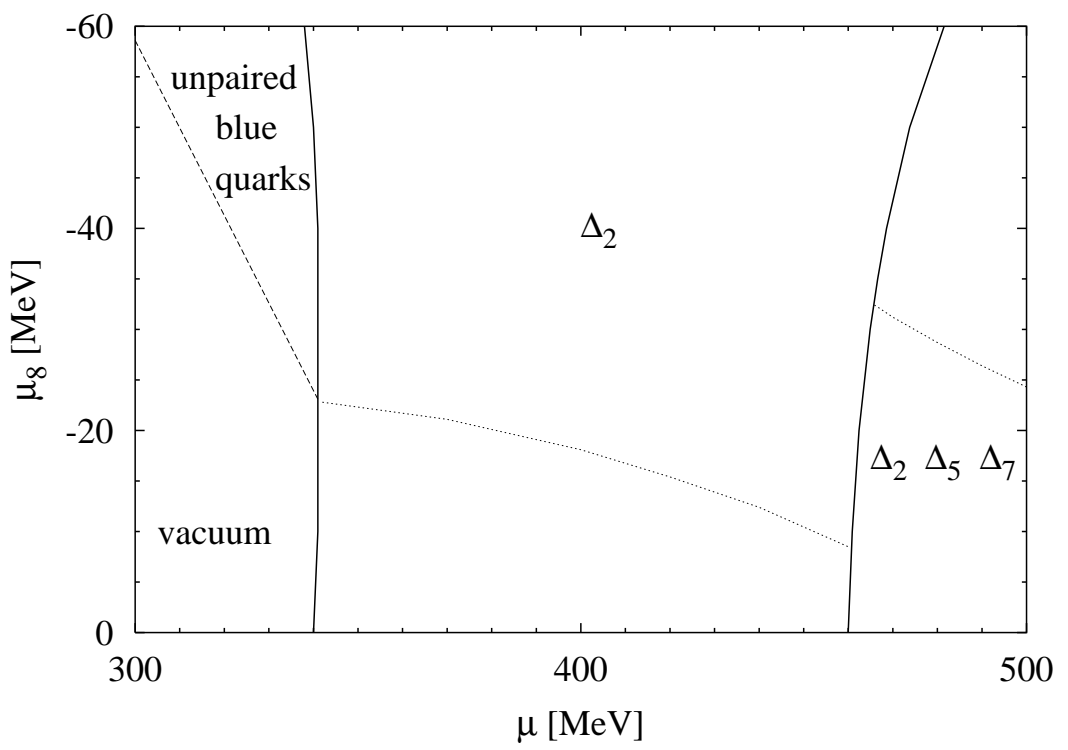

Figure 2: Phase diagram in the $\mu-\mu_{8}$ plane for $T=\mu_{3}=\mu_{Q}=0$. The various phases separated by the solid lines are characterized by different non-vanishing diquark gaps $\Delta_{i}$ as indicated in the figure. In the non-superconducting phase quarks are present only above the dashed line. The dotted lines are the lines of color neutral matter. In the CFL phase this also corresponds to electrically neutral matter.

In Fig. 图 we show the phase diagram in the $\mu-\mu_{Q}$-plane for $\mu_{8}=\mu_{3}=0$. Since we are interested in neutralizing the electrically positive $2 \mathrm{SC}$ phase, we choose $\mu_{Q}$ to be negative. As long as this is not too large, we find again the normal phase at lower values of $\mu$, the $2 \mathrm{SC}$ phase in the intermediate region and the CFL phase for large $\mu$. This changes dramatically around $\mu_{Q} \simeq 180 \mathrm{MeV}$ where both, the 2SC phase and the CFL phase disappear and a new phase emerges. This phase is analogous to the $2 \mathrm{SC}$ phase but with $d s$ pairing, instead of $u d$ pairing ( $2 \mathrm{SC}_{d s}$ "). In a small intermediate regime there is even another phase which contains us and $d s$ but no $u d$ pairs ("SC$u s+d s ")$.

Qualitatively, the existence of these phases is quite plausible: At low values of $\left|\mu_{Q}\right|$ the Fermi momenta of the up and down quarks are relatively similar to each other, whereas the strange quarks are suppressed because of their larger mass. With increasing negative $\mu_{Q}$, however, the up quarks become more and more disfavored and eventually the Fermi momenta are ordered as $p_{F}^{u}<p_{F}^{s}<p_{F}^{d}$. It is then easy to imagine that only $d s$ pairing or - in some intermediate regime - us and $d s$ pairing is possible.

Following this argument one might expect that there is always a value of $\mu_{Q}$, where the Fermi momenta of up and strange quarks are equal and hence the 2SC phase should be either followed by the CFL phase or by a phase with us-pairing only. However, this is not the case because of the discontinuous behavior of the quark masses. This is illustrated in Fig. 1 where the diquark gaps and constituent quark masses are shown as functions of $\mu_{Q}$ for fixed $\mu=390 \mathrm{MeV}$ and $\mu_{3}=\mu_{8}=0$. The $2 \mathrm{SC}-\mathrm{SC}_{u s+d s}$ phase transition takes place at $\mu_{Q}=-178.6 \mathrm{MeV}$, corresponding to $\mu_{u}=\mu+2 / 3 \mu_{Q} \simeq 270 \mathrm{MeV}$ and $\mu_{d}=$ $\mu_{s}=\mu-1 / 3 \mu_{Q} \simeq 450 \mathrm{MeV}$. Below the transition point the strange quark mass is even larger than $460 \mathrm{MeV}$ and, consequently, no strange quarks are present. At the transition 


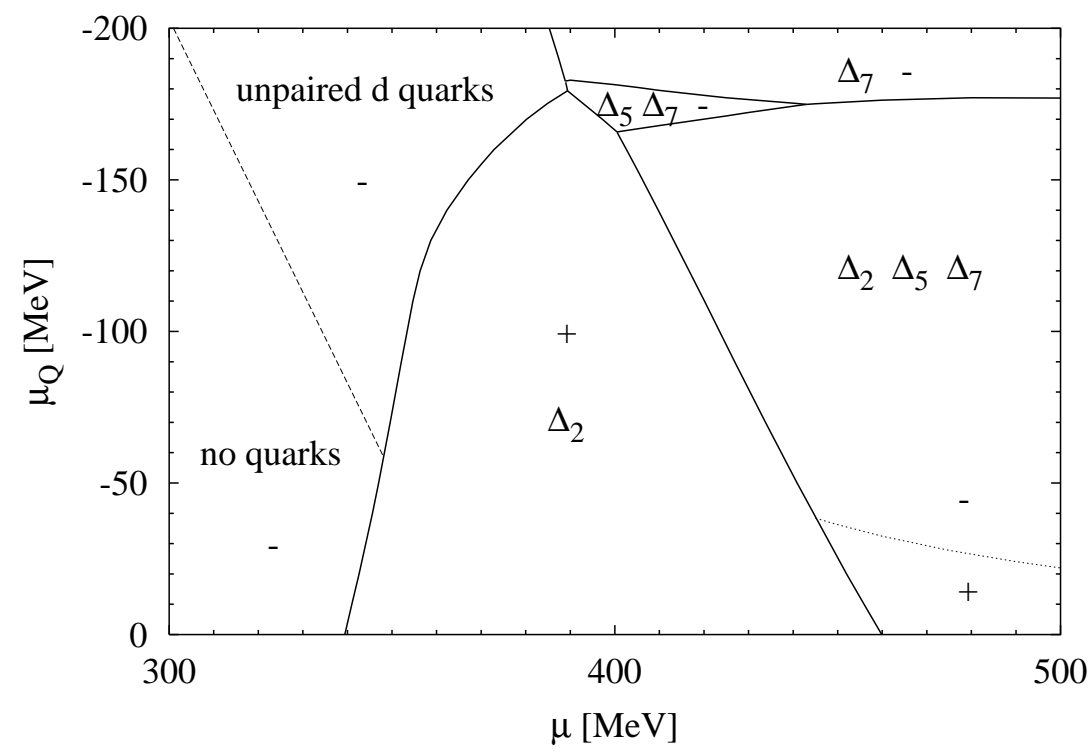

Figure 3: Phase diagram in the $\mu-\mu_{Q}$ plane for $T=\mu_{3}=\mu_{8}=0$. The various phases separated by the solid lines are characterized by different non-vanishing diquark gaps $\Delta_{i}$ as indicated in the figure. In the non-superconducting phase quarks are present only above the dashed line. The "+" and "-" signs indicate the sign of the electric charge density in the corresponding region. The dotted line corresponds to electrically (but not color) neutral matter in the CFL phase.

point the strange quark mass drops to $310 \mathrm{MeV}$ and the nominal Fermi momentum $p_{F}^{s}=\sqrt{\mu_{s}^{2}-M_{s}^{2}}$ is immediately larger than $p_{F}^{u}$.

The stability of the various condensates is rather well described by the criterion 24]

$$
\Delta_{i j} \gtrsim\left|\frac{p_{F}^{i}-p_{F}^{j}}{\sqrt{2}}\right|=: \Delta_{i j}^{c},
$$

where $\Delta_{i j}$ is the gap related to the pairing of the quark species $i$ and $j$. In the 2SC phase just below the phase boundary we have $\Delta_{2}=132.8 \mathrm{MeV}$, slightly larger than $\Delta_{2}^{c}=$ 127.4 MeV. At the phase boundary $\Delta_{2}^{c}$ rises to $133.6 \mathrm{MeV}$ due to a sudden increase of the up quark mass by more than $40 \mathrm{MeV}$. Taking the earlier value of $\Delta_{2}$, the above criterion is no longer fulfilled, which is consistent with our numerical result that the $u d$-pairs break up. This level of agreement is certainly better than one should expect. In fact, in the $\mathrm{SC}_{u s+d s}$ phase we find $\Delta_{5}$ continuously decreasing from $50.8 \mathrm{MeV}$ to $49.1 \mathrm{MeV}$ whereas $\Delta_{5}^{c}$ increases from $48.2 \mathrm{MeV}$ to $52.6 \mathrm{MeV}$, slightly violating Eq. (27). It is nevertheless possible to understand the break-up of the us pairs, which occurs at $\mu_{Q}=-183.0 \mathrm{MeV}$, from the fact that at this point $\Delta_{5}^{c}$ jumps to $62.6 \mathrm{MeV}$ due to a further increase of $M_{u}$ and a further decrease of $M_{s}$. Moreover, the fact that we always find $\Delta_{5} \approx \Delta_{5}^{c}$, at least in this example, indicates that the $\mathrm{SC}_{u s+d s}$ phase is rather fragile and might disappear upon small variations of the model parameters.

In the phase diagram, Fig. 3, we also indicated the sign of the electric charge density for the various regions, and the line of electrically neutral matter in the CFL phase (dotted line). Note that there is no other electrically neutral regime in this diagram (apart from 

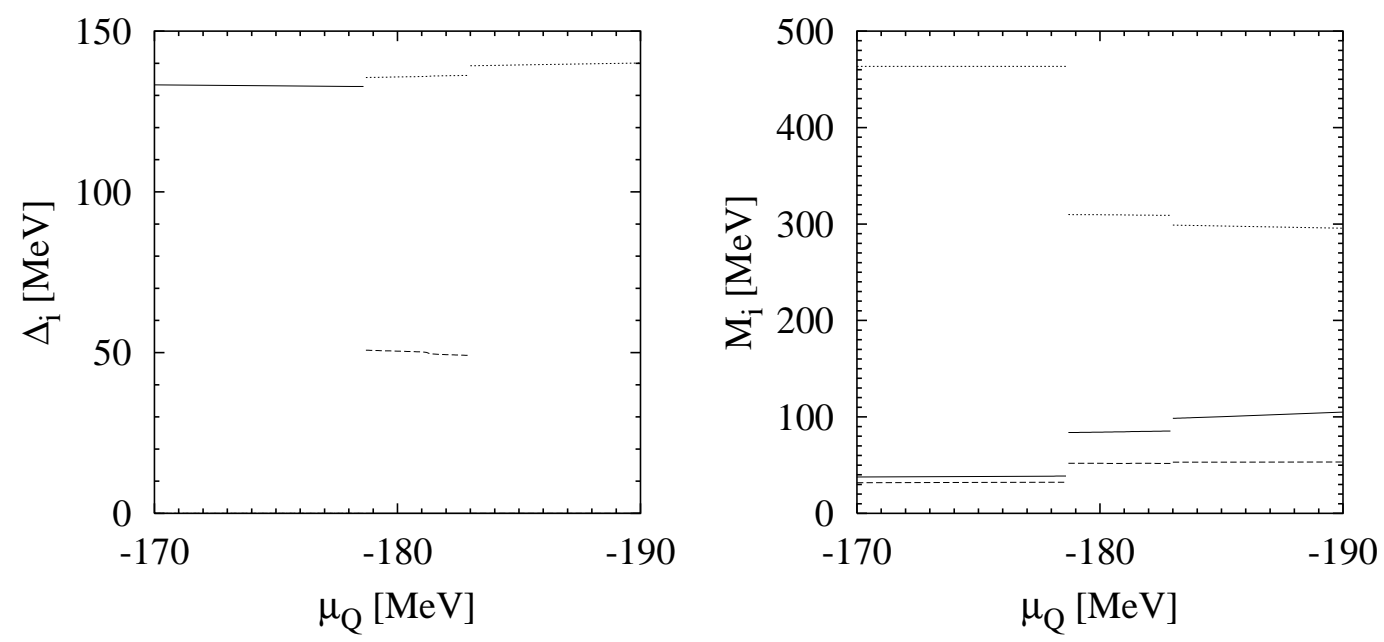

Figure 4: Diquark gaps and quark masses for $T=\mu_{3}=\mu_{8}=0$, and $\mu=390 \mathrm{MeV}$ as functions of $\mu_{Q}$. Left: $\Delta_{2}$ (solid), $\Delta_{5}$ (dashed), and $\Delta_{7}$ (dotted). Right: $M_{u}$ (solid), $M_{d}$ (dashed), and $M_{s}$ (dotted).

the vacuum at small $\mu$ and $\mu_{Q}=0$ ). In the normal phase, there are again no quarks below the dashed line, corresponding to the line $\mu-1 / 3 \mu_{Q}=M_{d}$. This region is nevertheless negatively charged due to the leptons which are present for any $\mu_{Q}<0$. Above the dashed line there are also $d$ quarks rendering the matter even more negative. (In the right corner of this phase there is also a very small fraction of $u$ quarks.)

The "new" phases, $2 \mathrm{SC}_{d s}$ and $\mathrm{SC}_{u s+d s}$, are also negatively charged. On the contrary, the entire 2SC phase is positively charged, even at the largest values of $\left|\mu_{Q}\right|$. This is illustrated in Fig. 5 where the various charge densities $n_{i}$ divided by the total quark number density $n$ are plotted as functions of $\mu_{Q}$, again for fixed $\mu=390 \mathrm{MeV}$ and $\mu_{3}=\mu_{8}=0$. As expected, $n_{Q} / n$ (solid line) decreases with increasing negative $\mu_{Q}$. However, in the $2 \mathrm{SC}$ phase $\left(0 \geq \mu_{Q}>-178.6 \mathrm{MeV}\right)$ it stays positive and before the point of neutrality is reached the phase transition to the $\mathrm{SC}_{u s+d s}$ phase takes place.

The difficulty to obtain electrically neutral $2 \mathrm{SC}$ matter can be traced back to the fact that according to Eq. (26), the sum of red and green $u$ quarks is equal to the sum of red and green $d$ quarks. As long as no strange quarks are present, the related positive net charge can only be compensated by the blue quarks and the leptons, which would require a very large negative $\mu_{Q}$. However, before this point is reached it becomes more favorite to form a different phase with a relatively large fraction of strange quarks which then also

\begin{tabular}{|c|c|c|c|c|c|c|c|c|}
\hline phase & $\mathrm{N}$ & $2 \mathrm{SC}$ & $2 \mathrm{SC}_{u s}$ & $2 \mathrm{SC}_{d s}$ & $\mathrm{SC}_{u d+u s}$ & $\mathrm{SC}_{u d+d s}$ & $\mathrm{SC}_{u s+d s}$ & $\mathrm{CFL}$ \\
\hline diquark gaps & - & $\Delta_{2}$ & $\Delta_{5}$ & $\Delta_{7}$ & $\Delta_{2}, \Delta_{5}$ & $\Delta_{2}, \Delta_{7}$ & $\Delta_{5}, \Delta_{7}$ & $\Delta_{2}, \Delta_{5}, \Delta_{7}$ \\
\hline
\end{tabular}

Table 1: Phases and corresponding non-vanishing diquark gaps. 


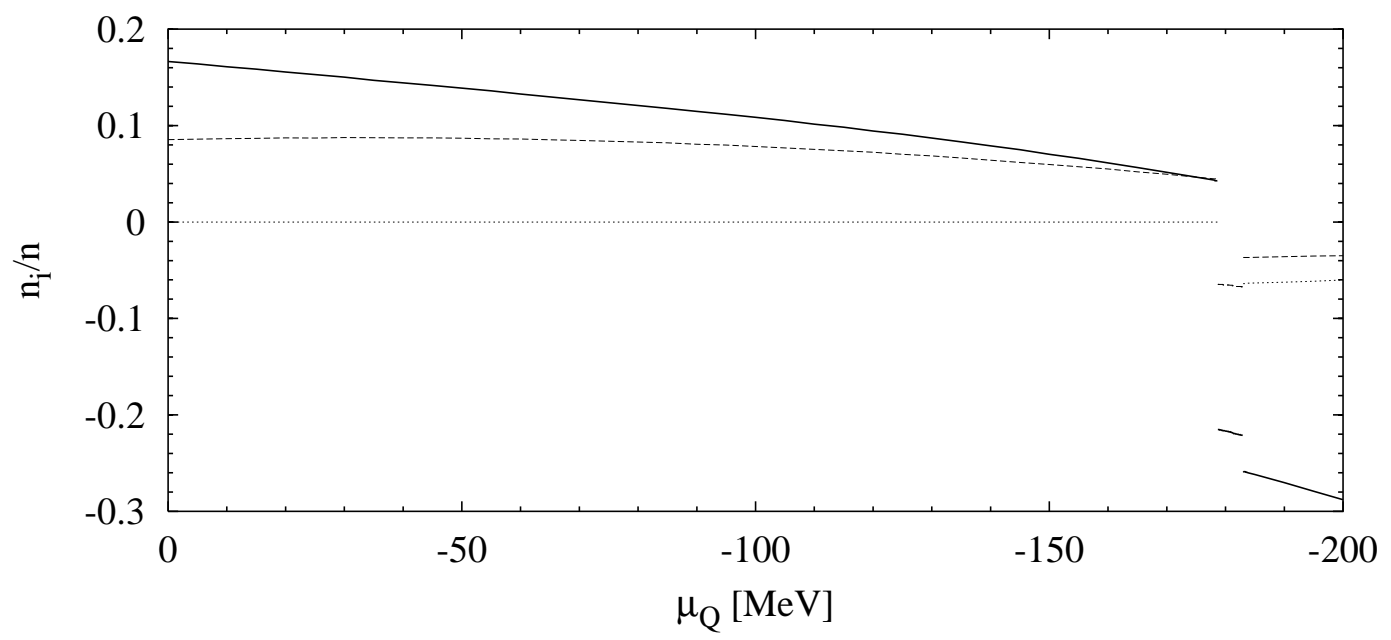

Figure 5: Relative densities for $T=\mu_{3}=\mu_{8}=0$, and $\mu=390 \mathrm{MeV}$ as functions of $\mu_{Q}: n_{Q} / n$ (solid), $n_{8} / n$ (dashed), and $n_{3} / n$ (dotted).

participate in a diquark condensate. As discussed above, the selfconsistent treatment, which leads to a sudden drop of the strange quark mass and hence to a sudden increase of the strange Fermi momentum, is crucial in this context.

In the above discussion we have not considered the effect of a non-vanishing chemical potential $\mu_{8}$ on top of a non-vanishing $\mu_{Q}$. Since the blue quarks are the main carriers of negative electric charge in the 2SC phase, one could hope that increasing the number of blue quarks, as necessary for color neutrality, could also help to electrically neutralize 2SC matter. It turns out, however, that the rather small values of $\mu_{8}$ which are needed for color neutrality (see Ref. [12] and Fig. 2) do not change the above results qualitatively.

So far we always kept $\mu_{3}=0$. In fact, there is no need to vary $\mu_{3}$, as long as we are mainly interested in finding electrically and color neutral solutions of homogeneous normal, 2SC, or CFL matter. However, as we will see in the next section, the construction of neutral mixed phases requires also non-vanishing values of $\mu_{3}$. In this context we will encounter another phase, which is not present in Figs. 2 and 3 . For illustration we consider a plane in the four-dimensional $\left\{\mu_{i}\right\}$-space where $\mu$ and $\mu_{Q}$ are taken as independent variables and $\mu_{3}$ and $\mu_{8}$ are given by $\mu_{3}=-\mu_{Q} / 2$ and $\mu_{8}=-\mu_{Q} / 7-30 \mathrm{MeV}$. The relevance of this particular choice will become more clear in the next section. Here we just note that $\mu_{3}=-\mu_{Q} / 2$ means that $\mu_{u, r}=\mu_{d, g}$. Also the sum $\mu_{s, r}+\mu_{u, b}$, corresponding to the chemical potential related to a pair of a red strange quark and a blue up quark, equals the sum $\mu_{s, g}+\mu_{d, b}$, corresponding to the chemical potential related to a pair of green strange quarks and blue down quarks. Together with the relations given above Eq. (25) and the isospin symmetry of the original Lagrangian this implies for the CFL phase that $n_{u}=n_{d}$ or, equivalently, $n_{r}=n_{g}$ and thus $n_{3}=0$.

In Fig. 6 we show a small part of the resulting phase diagram. Here, in addition to the standard 2SC and CFL phases, we find a phase where only $u$ and $s$ quarks are paired

\footnotetext{
$\S$ This is very similar to the arguments of Alford and Rajagopal 10. The main difference is that we do not compare different neutral phases with each other, but phases in chemical equilibrium.
} 


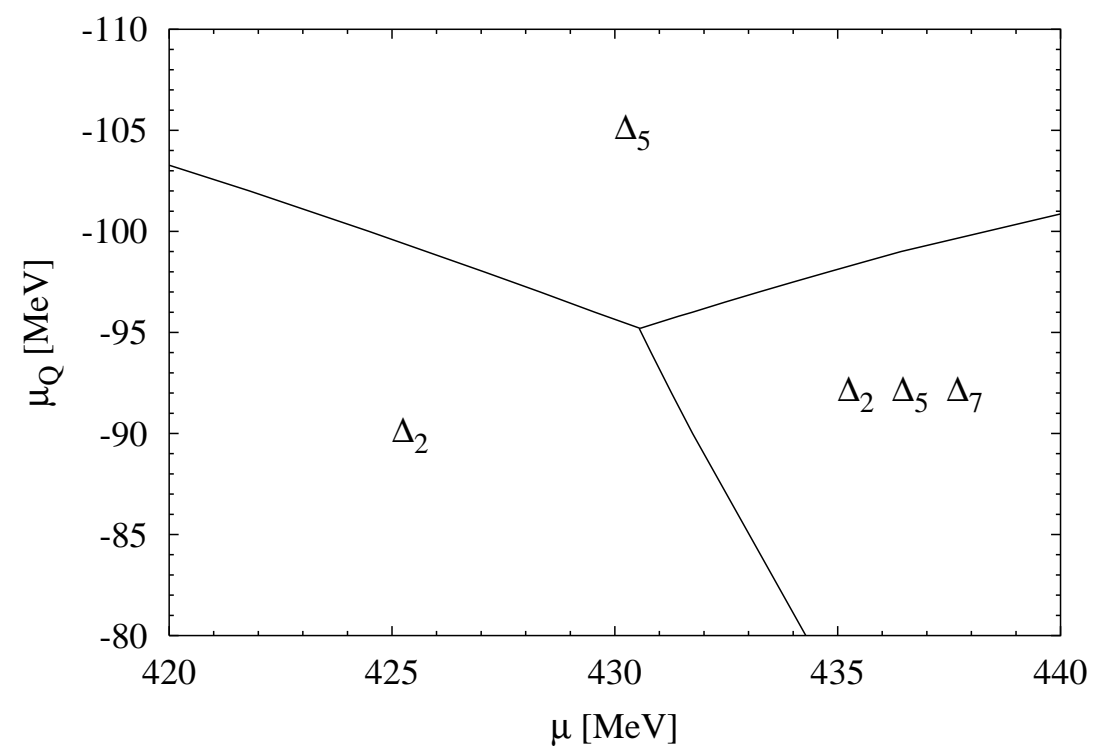

Figure 6: Phase diagram in a plane defined by $\mu$ and $\mu_{Q}$ as independent variables and $\mu_{3}=-\mu_{Q} / 2$ and $\mu_{8}=-\mu_{Q} / 7-30 \mathrm{MeV}$. The various phases separated by the solid lines are characterized by different non-vanishing diquark gaps $\Delta_{i}$ as indicated in the figure.

(" $2 \mathrm{SC}_{u s}$ "). This means, we have found examples for all three $\Delta_{A}, A=2,5,7$, being the only non-vanishing scalar diquark gaps in some regime. Taking all possible combinations of no, one, two, or three of these condensates (see Table 1), the phases $\mathrm{SC}_{u d+u s}$ and $\mathrm{SC}_{u d+d s}$, i.e., the combinations $\Delta_{2}+\Delta_{5}$ and $\Delta_{2}+\Delta_{7}$ are the only ones we have not encountered. These phases might exist as well, but we did not perform a systematic investigation of this question, which was not the goal of this work.

\section{$5 \quad$ Mixed phases}

Having gained some overview about the general phase structure of our system we are now in the position to construct electrically and color neutral mixed phases. Our starting point is $\mu=465.7 \mathrm{MeV}, \mu_{8}=-32.5 \mathrm{MeV}$, and $\mu_{3}=\mu_{Q}=0$ where the line of neutral CFL matter meets the boundary to the $2 \mathrm{SC}$ phase. At lower values of $\mu$, mixed phases become possible and are energetically favored as long as Coulomb and surface effects are neglected. Following the guidelines worked out in Sec. 2 we find nine regimes characterized by different compositions of coexisting phases (see Table 2). The corresponding chemical potentials $\mu_{i}$ as functions of $\mu$ are displayed in Fig. 0. In Table 2 we also list the corresponding minimal and maximal quark number densities, averaged over the components of the respective mixed phase.

In the regime closest to the region of homogeneous neutral CFL matter $(430.6 \mathrm{MeV}<$ $\mu<465.7 \mathrm{MeV}$ ), we find a mixed phase consisting of a CFL component and a $2 \mathrm{SC}$ component. The volume fraction $x_{2 S C}$ of the $2 \mathrm{SC}$ component is displayed in the left panel of Fig. 8. In the higher- $\mu$ part of this region it is completely negligible, but even at the lower end it remains below $2 \%$. Consequently, the CFL component must stay almost 
neutral by itself. Indeed, the relative charge densities $n_{i} / n, i=3,8, Q$, (right panel of Fig. 8) are very small. As we have discussed in the previous section, $n_{3}$-neutrality of the CFL phase is maintained by the relation $\mu_{3}=-\mu_{Q} / 2$. For the actual values of $\mu_{3}$ and $\mu_{Q} / 2$ in the 2SC-CFL mixed phase we find a deviation of less than $1 \%$ from this relation, while $\mu_{8}$ can approximately be fitted by $\mu_{8}=-\mu_{Q} / 7-30 \mathrm{MeV}$. This is the reason why we have calculated the phase diagram shown in Fig. 6 with these constraints.

As can be seen there, the 2SC-CFL phase boundary meets the boundary to the $2 \mathrm{SC}_{u s}$ phase at $\mu=430.6 \mathrm{MeV}$ and we get a three-component neutral mixed phase, consisting of 2SC, CFL and $2 \mathrm{SC}_{u s}$. Below that, on a short interval in $\mu$, we even find a four-component neutral mixed phase (2SC, CFL, $2 \mathrm{SC}_{u s}$, and $\mathrm{SC}_{u s+d s}$ ) before upon further decreasing $\mu$ the system goes over into a neutral $2 \mathrm{SC}-\mathrm{CFL}-\mathrm{SC}_{u s+d s}$ mixed phase.

In Fig. 9 the volume fractions of the various components of the mixed phases are plotted as functions of $\mu$. Whereas the 2SC-CFL-mixed phase (Fig. 8) is completely dominated by the CFL component, thereafter the CFL fraction becomes quickly smaller with decreasing $\mu$, while in particular the $2 \mathrm{SC}$ component, becomes more and more important. At $\mu=407.7 \mathrm{MeV}$ the CFL component disappears completely.

An admixture of normal quark matter is found below $\mu=388.8 \mathrm{MeV}$. The fractions of the superconducting phases other than the 2SC phase then rapidly become smaller and vanish at $\mu=388.6 \mathrm{MeV}$, while the fraction of normal matter strongly increases. I" Nevertheless the $2 \mathrm{SC}$ phase stays the dominant component for $\mu \gtrsim 360 \mathrm{MeV}$.

As discussed in the previous section, apart from the vacuum there is no region of stable neutral non-superconducting quark matter. Therefore the normal-2SC mixed phase cannot end in normal homogeneous quark matter but only in the vacuum. To that end, the chemical potential $\mu_{Q}$ must finally go to zero. Eventually, at $\mu=348.6 \mathrm{MeV},\left|\mu_{Q}\right|$ drops below $60 \mathrm{MeV}$ and we enter the regime where the normal phase only consists of electrons without quarks (cf. Fig. 3). This means the corresponding mixed phase consists of (electrically positive) 2SC-droplets surrounded by regions without quarks and neutralized

INote, however, that all volume fractions $x_{i}$ are continous functions in the entire mixed phase region.

\begin{tabular}{|l|c|c|}
\hline components & $\mu[\mathrm{MeV}]$ & $n\left[\mathrm{fm}^{-3}\right]$ \\
\hline $\mathrm{N}, 2 \mathrm{SC}$ & $340.9-388.6$ & $0.00-1.56$ \\
\hline $\mathrm{N}, 2 \mathrm{SC}, \mathrm{SC}_{u s+d s}$ & $388.6-388.7$ & $1.56-1.56$ \\
\hline $\mathrm{N}, 2 \mathrm{SC}, \mathrm{SC}_{u s+d s}, 2 \mathrm{SC}_{u s}$ & $388.7-388.8$ & $1.56-1.56$ \\
\hline $2 \mathrm{SC}, \mathrm{SC}_{u s+d s}, 2 \mathrm{SC}_{u s}$ & $388.8-395.4$ & $1.56-1.74$ \\
\hline $2 \mathrm{SC}, \mathrm{SC}_{u s+d s}$ & $395.4-407.7$ & $1.74-2.59$ \\
\hline $2 \mathrm{SC}, \mathrm{SC}_{u s+d s}, \mathrm{CFL}$ & $407.7-426.5$ & $2.59-2.90$ \\
\hline $2 \mathrm{SC}, \mathrm{SC}_{u s+d s}, \mathrm{CFL}, 2 \mathrm{SC}_{u s}$ & $426.5-427.1$ & $2.90-2.93$ \\
\hline 2SC, CFL, $2 \mathrm{SC}_{u s}$ & $427.1-430.6$ & $2.93-3.11$ \\
\hline 2SC, CFL & $430.6-465.7$ & $3.11-3.92$ \\
\hline
\end{tabular}

Table 2: Composition of electrically and color neutral mixed phases, corresponding quark number chemical potentials and average quark number densities. The various components are defined in Table 1. 


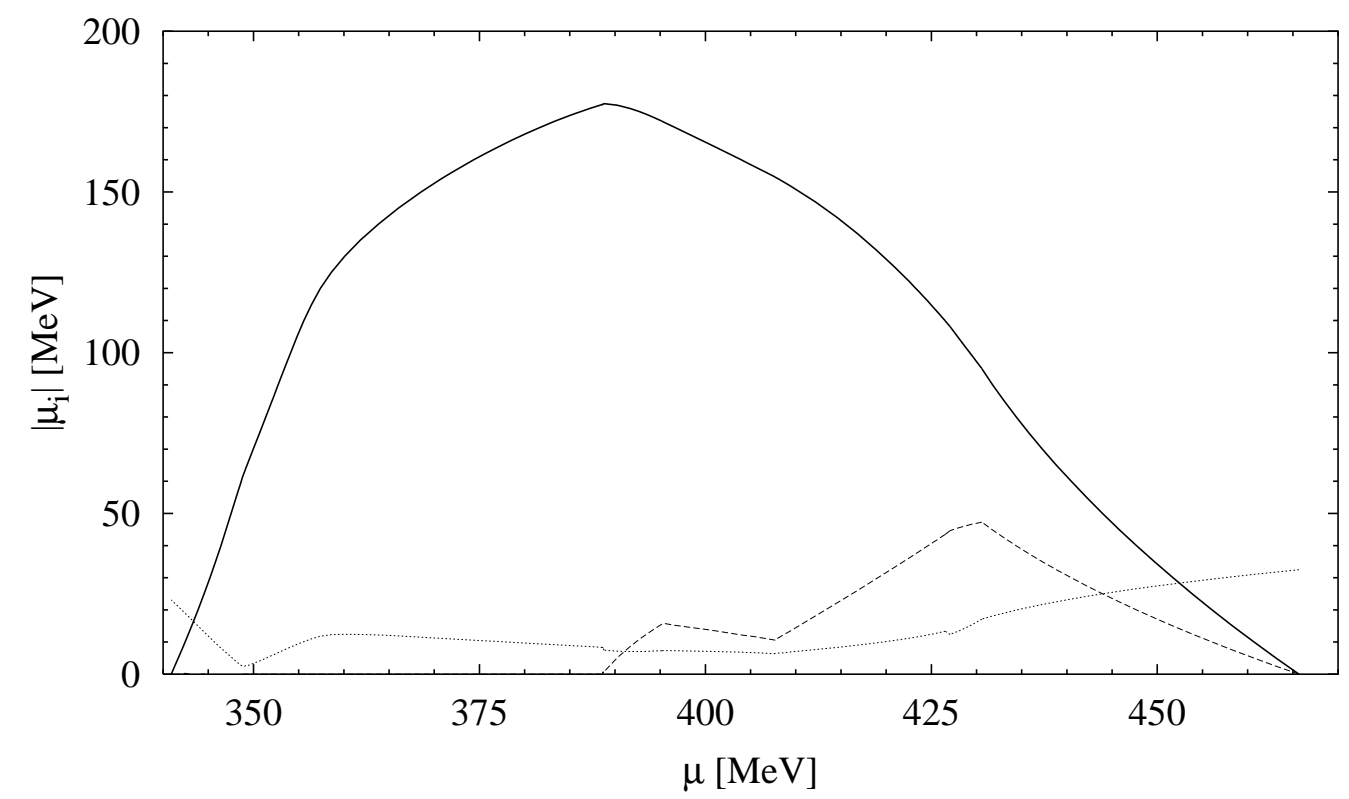

Figure 7: Chemical potentials $\mu_{i}$ corresponding to the electrically and color neutral mixed phases, listed in Table 2: $-\mu_{Q}$ (solid), $\mu_{3}$ (dashed), and $-\mu_{8}$ (dotted).
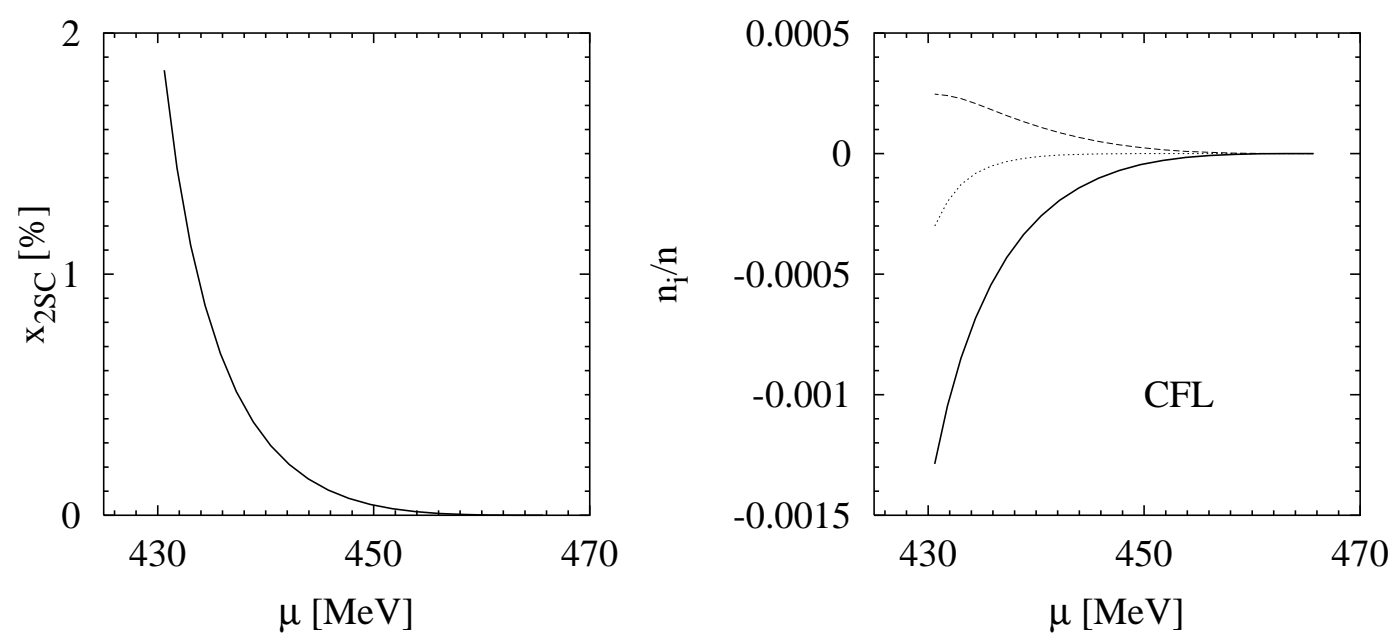

Figure 8: Quantities related to the neutral 2SC-CFL mixed phase as functions of the quark number chemical potential $\mu$. Left: Volume fraction $x_{2 S C}$ of the 2SC component. Right: Relative densities in the CFL component: $n_{Q} / n$ (solid), $n_{8} / n$ (dashed), and $n_{3} / n$ (dotted). 


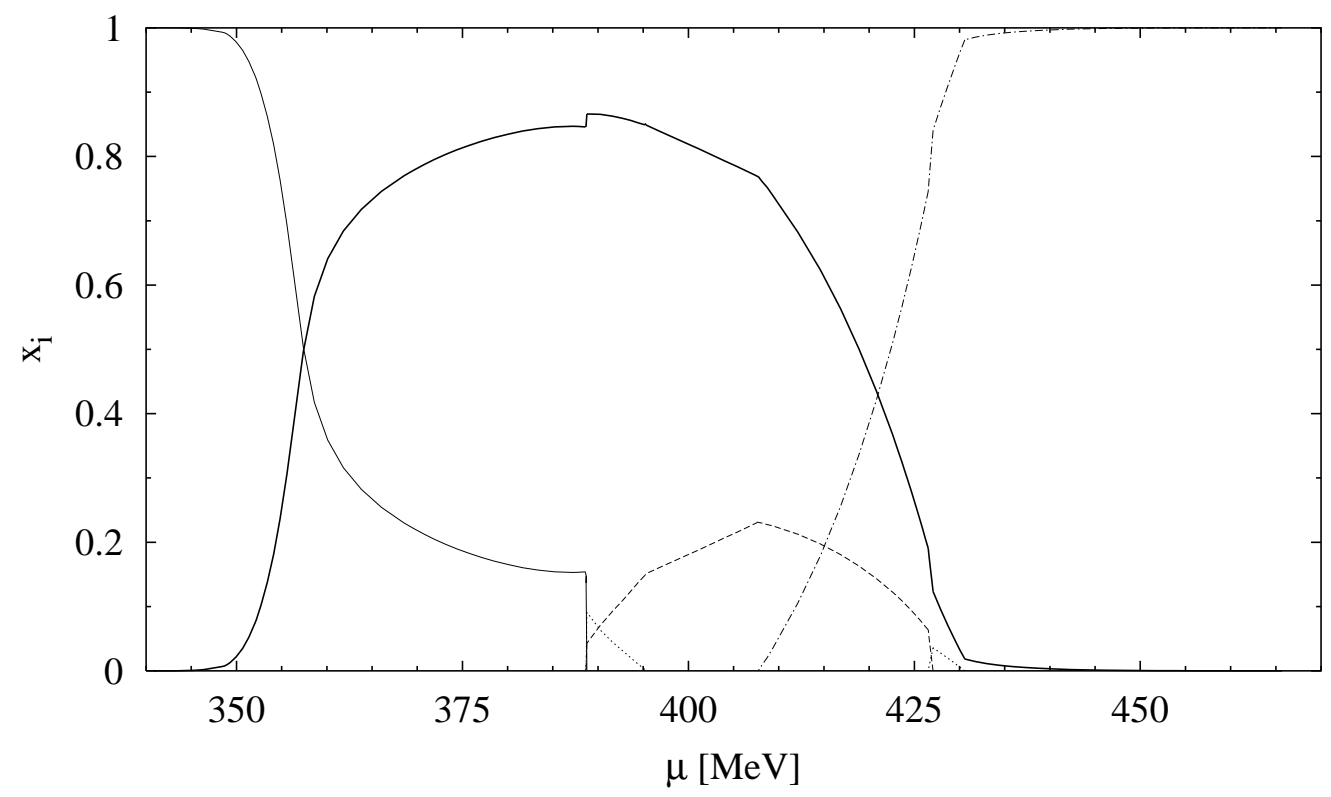

Figure 9: Volume fractions of the various components in the mixed phase region as functions of $\mu$ : normal (thin solid), 2SC (thick solid), CFL (dashed-dotted), $\mathrm{SC}_{u s+d s}$ (dashed), $2 \mathrm{SC}_{u s}$ (dotted).

by a homogeneous background of electrons. Since the electrons are color neutral, the 2SC component must be color neutral by itself. This is maintained by an increase of $\left|\mu_{8}\right|$ in this regime. At $\mu=340.9 \mathrm{MeV}$ we finally reach the vacuum.

Qualitatively our results are in agreement with those obtained in Ref. [12], where no mixed phase was admitted. The regions which are dominated by the CFL phase and the 2SC phase, respectively, correspond roughly to those values of the chemical potential where the authors of Ref. [12 find that the CFL or the 2SC phase, respectively, is favored. Of course, since we started from the same Lagrangian, these homogeneous neutral solutions also exist in our model. We find, for instance, below $\mu=465.7 \mathrm{MeV}$ a color and electrically neutral CFL phase which is, however, less favored than the 2SC-CFL mixed phase. At this point we should recall that in our calculations we have neglected the surface energy and the energies of the electric and color-electric fields. Clearly the mixed phases are only stable if the gain in bulk free energy is larger than these so-far neglected contributions. If this is not the case we recover the results of Ref. [12]. In a neutron star we would then find homogeneous phases separated by sharp interfaces.

For the two-component mixed phases the surface and Coulomb contributions can be estimated along the lines of the analysis performed in Ref. [14] for the interface between nuclear and CFL matter. Because of the smallness of the 2SC fraction in the 2SC-CFL mixed phase the gain in bulk free energy compared with a pure CFL phase is extremely small in this case (less than $0.1 \mathrm{MeV} / \mathrm{fm}^{3}$ ). It is therefore more or less obvious that this mixed phase will not be stable. The situation looks a little more promising for the 2SC-SC $\mathrm{SC}_{u s+d s}$-phase at $\mu \approx 400 \mathrm{MeV}$ where the rarer phase, i.e., the $\mathrm{SC}_{u s+d s}$-phase, is more populated. Here we find a gain in bulk energy of about $4 \mathrm{MeV} / \mathrm{fm}^{3}$. However, even in this case a calculation along the lines of Ref. [14] shows that this gain is weight out 
by Coulomb and surface energy already for relatively small values of the surface tension $\sigma \approx 10 \mathrm{MeV} / \mathrm{fm}^{2}$. Note that the true value of $\sigma$ is an unknown quantity which does not follow from our model. However, as argued in Ref. [14 it is likely to be much larger than the above value.

For mixed phases with three or four components the situation is obviously more complicated. It seems, however, rather unlikely that an admixture of more than two components can be favored if the surface and Coloumb energy already inhibits the existence of a phase with two components. The more components we have the more contributions to surface, Coulomb and color charge energy we have. On the other hand, even if, e.g., a 3-component mixed phase turns out to be unstable, we cannot exclude to find a stable 2-component mixed phase instead. A definite answer to these questions lies beyond the scope of this paper and we postpone it to future work.

\section{Summary and Discussion}

We performed an investigation of quark matter under conditions which possibly occur in the interior of neutron stars older than a few minutes, i.e., dense, electrically and color neutral matter in $\beta$-equilibrium. Recently it was shown by Alford and Rajagopal that these constraints have important effects, in particular that they might disfavor the standard two-flavor color superconducting phase (2SC) in neutron stars [10]. In the present article we focussed on the formation of mixed phases, i.e., different coexisting phases in chemical equilibrium. In this case the neutrality conditions have only to be fulfilled globally [13].

Our model calculations are performed within an NJL-type model, which allows to treat diquark condensates on an equal footing with quark-antiquark condensates, leading to density (and phase-) dependent effective quark masses [7]. In accordance with our expectations the regimes of lower or higher quark number chemical potentials are dominated by normal (non-superconducting) matter and by matter in the color-flavor locked (CFL) phase, respectively. In addition, we find a certain regime of quark number chemical potentials where the 2SC phase is the dominant component. Naively one would expect that the above three phases represent the only constituents of the mixed phases we encounter. However, in addition we find admixtures of more exotic phases, like, e.g., a $\mathrm{SC}_{u s+d s}$-phase or a $2 \mathrm{SC}_{u s}$-phase. These phases occur because the chemical potentials, in particular $\mu_{Q}$, necessary to enforce electric neutrality, are often large enough such that the ordering of the Fermi momenta is reversed: $p_{F}^{u}<p_{F}^{s}<p_{F}^{d}$.

Simple estimates show that the gain in bulk energy due to the formation of these mixed phases is probably not large enough to compensate for the energy arising from surface, and electric and color charge effects. This seems to indicate that homogeneous phases of neutral quark matter separated by sharp interfaces are favored. This could include the possibility of a stable neutral 2SC phase. However, a more careful analysis is certainly needed before a definite answer to this question can be given. There are other aspects which deserve to be studied more closely in future:

At low densities we certainly need a more realistic description of the hadronic phase, which in the present model at best is represented by a vacuum or quark phase with 
spontaneously broken chiral symmetry. A more complete calculation, including a realistic equation of state of nuclear matter, has to show whether in particular the N-2SC-phase which we find at rather low densities is robust.

At higher densities we should also take into account the possibility of a CFL-phase with kaon condensation [29, 30]. Recently the authors of Ref. [12] have estimated the effect of this possibility on the equation of state of homogeneous neutral quark matter. They found that the kaon condensate lowers the critical quark chemical potential for the 2SC-CFL phase transition by about $16 \mathrm{MeV}$. This is not dramatic but could make a difference if for instance the transition point to the hadronic phase turns out to be in the same region.

We also neglected the possibility of the formation of crystalline (LOFF) phases [15, 27]. These become eventually favored over phases with BCS pairing if the Fermi surfaces are pulled apart. As we are mostly dealing with pairing of quarks with unequal Fermi momenta, we suppose that especially in the vicinity of phase boundaries, the possible formation of a crystalline phase should be taken into account. In fact, the occurrence of mixed phases which are necessarily related to non-uniform structures in space already points into this direction. The neglect of gradient terms in our mean-field approach is only justified if the structures are much larger than the average distance between the quarks. Unfortunately, we cannot say much about the sizes in the mixed phases as long as we do not know the surface tension. However, taking relatively small surface tensions as required to keep the mixed phases energetically stable, the sizes of the rarer components are typically only a few times larger than the inter-quark distances.

Recently another interesting pairing configuration has been discussed by Liu and Wilczek 28]. A so-called "interior gap" can be formed in systems with unequal Fermi momenta where the Fermi momentum of the heavier fermion species is larger than that of the lighter species. The authors of Ref. [28] claim that the "interior gap" configuration is favored if

$$
1>\frac{p_{F}^{a}+p_{F}^{b}}{2 p_{F}^{a}} \frac{M_{a}}{M_{a}+M_{b}},
$$

where $M_{a}<M_{b}$ and $p_{F}^{a}<p_{F}^{b}$. Usually this possibility can be excluded in studies of superconducting quark matter, since the Fermi momentum of the heavier strange quarks is smaller than the Fermi momenta of up and down quarks. But, as we have discussed above, the existence of "exotic" phases, like, e.g., the $\mathrm{SC}_{u s+d s}$-phase, can be traced back to the fact that the Fermi momenta are ordered as $p_{F}^{u}<p_{F}^{s}<p_{F}^{d}$. Therefore, uspairing in the "interior gap" configuration seems possible. Let us look at some numerical example: At $\mu \approx 400 \mathrm{MeV}$ we find $\mathrm{SC}_{u s+d s}$ matter with $M_{u} \approx 60 \mathrm{MeV}, M_{s} \approx 300 \mathrm{MeV}$, $p_{F}^{u} \approx 285 \mathrm{MeV}$, and $p_{F}^{s} \approx 350 \mathrm{MeV}$. This leads to values of about 0.2 for the right hand side of Eq. (28). Thus it seems probable that such a phase occurs.

It has also to be mentioned that we have restricted ourselves to diquark condensates $s_{A A^{\prime}}$ with $A=A^{\prime}$. Originally this was motivated by the fact that, e.g., in the 2SC phase any linear combination of $s_{22}, s_{25}$, and $s_{27}$ can be rotated into the 22-direction by a global color transformation. However, in principle we can always construct a color neutral mixed phase without applying color chemical potentials by combining several components of the same condensate but rotated into different color directions. Within our present formalism 
this would further lower the free energy, but it is rather unlikely, whether this is still the case when the color electric energy of the various domains are taken into account. Anyway, problems, like the difficulty to find stable 2SC matter, mostly arise from the constraint of electric neutrality, whereas imposing color neutrality is only a minor effect.

Note that we only considered the situation relevant for neutron stars older than a few minutes, i.e., when the neutrinos can freely leave the star. The authors of Ref. [12] performed an extended analysis looking also at a system at finite temperature and lepton content. These complications are likely to be relevant during the evolution of the star from a proto-neutron star at high temperature where neutrinos are trapped to a cold compact star where neutrinos can freely leave the star. Steiner, Reddy and Prakash [12] showed that in this case the 2SC phase is favored because the CFL phase excludes electrons and can therefore not easily accommodate a finite lepton number chemical potential. A study of mixed phases in this context is left to future work.

\section{Acknowledgments}

Two of us (M.B. and M.O.) would like to thank K. Rajagopal for illuminating discussions and the ITP, Santa Barbara, for their hospitality and financial support (Grant No. PHY94-07194). M.O. acknowledges financial support from the Alexander von Humboldtfoundation as a Feodor-Lynen fellow.

\section{References}

[1] M. Alford, K. Rajagopal, and F. Wilczek, Phys. Lett. B 422 (1998), 247.

[2] R. Rapp, T. Schäfer, E.V. Shuryak, and M. Velkovsky, Phys. Rev. Lett. 81 (1998) 53.

[3] R. Rapp, T. Schäfer, E.V. Shuryak, and M. Velkovsky, Annals Phys. 280 (2000) 35.

[4] M. Alford, K. Rajagopal, and F. Wilczek, Nucl. Phys. B 537 (1999) 443.

[5] K. Rajagopal and F. Wilczek, hep-ph/0011333, and references therein.

[6] M. Alford, Ann. Rev. Nucl. Part. Sci. 51 (2001) 131.

[7] M. Buballa and M. Oertel, Nucl. Phys. A 703 (2002) 770.

[8] M. Oertel and M. Buballa, in: Ultrarelativistic heavy ion collisions, Proc. Intl. workshop XXX on gross properties of nuclei and nuclear excitations, Hirschegg/Austria, M. Buballa, W. Nörenberg, B.-J. Schaefer, and J. Wambach (eds.), Darmstadt (2002).

[9] P. Amore, M.C. Birse, J.A. McGovern, and N. R. Walet, Phys. Rev. D 65 (2002) 074005.

[10] M. Alford and K. Rajagopal, JHEP 0206 (2002) 031.

[11] M. Buballa and M. Oertel, Phys. Lett. B 457 (1999) 261.

[12] A. Steiner, S. Reddy, and M. Prakash, hep-ph/0205201.

[13] N.K. Glendenning, Phys. Rev. D 46 (1992) 1274. 
[14] M. Alford, K. Rajagopal, S. Reddy, and F. Wilczek, Phys. Rev. D 64 (2001) 074017.

[15] J. Bowers and K. Rajagopal, Phys. Rev. D 66 (2002), 065002.

[16] M. Takizawa, K. Tsushima, Y. Kohyama and K. Kubodera, Nucl. Phys. A 507 (1990) 611.

[17] S. Klimt, M. Lutz, U. Vogl and W. Weise, Nucl. Phys. A 516 (1990) 429;

U. Vogl, M. Lutz, S. Klimt and W. Weise, Nucl. Phys. A 516 (1990) 469.

[18] M. Lutz, S. Klimt and W. Weise, Nucl. Phys. A 542 (1992) 521.

[19] P. Rehberg, S.P. Klevansky and J. Hüfner, Phys. Rev. C 53 (1996) 410.

[20] M. Alford, J. Berges, K. Rajagopal, Nucl. Phys. B 558 (1999) 219.

[21] N. Ishii, W. Bentz, and K. Yazaki, Phys. Lett. B 318 (1993) 26.

[22] C. Hanhart and S. Krewald, Phys. Lett. B 344 (1995) 55.

[23] M. Buballa, J. Hošek, and M. Oertel, Phys. Rev. D 65 (2002) 014018.

[24] K. Rajagopal and F. Wilczek, Phys. Rev. Lett. 86 (2001), 3492.

[25] T. Schäfer, Phys. Rev. D 62 (2000) 094007.

[26] M. Buballa, J. Hošek, and M. Oertel, hep-ph/0204275.

[27] M. Alford, J. Bowers, and K. Rajagopal, Phys. Rev. D 63 (2001) 074016.

[28] W. V. Liu and F. Wilczek, cond-mat/0208052.

[29] P.F. Bedaque and T. Schäfer, Nucl. Phys. A 697 (2002) 802.

[30] D.B. Kaplan and S.Reddy, Phys. Rev. D 65 (2002) 054042. 